\title{
Posttraumatic Chylous Knee Effusion
}

Berber de Boer, MD, Robbert J. Goekoop, MD, Hagaziekenhuis, Rheumatology, Den Haag, the Netherlands. Address correspondence to Dr. B. de Boer, Albinusdreef 2, 2600 RC Leiden, the Netherlands. Email: b.de_boer@lumc.nl. The authors declare no conflicts of interest. Ethics board approval was not required according to the authors' institution. Patient's written informed consent to publish the material was obtained.

This is a very rare case of posttraumatic chylous joint effusion that has been described only a few times before in the literature. ${ }^{1,2,3,4,5}$

A 64-year-old White woman presented at the outpatient clinic with persistent knee pain for 5 weeks that she related to a fall. Of note in her medical history were limited cutaneous systemic sclerosis, osteoarthritis, and chondrocalcinosis of both knees, and a left patella reimplantation 35 years ago. There was no previous history of inflammatory arthritis. On physical examination, she had normal vital signs. There was painful swelling of the left suprapatellar bursa, without warmth or erythema. Range of motion in the knee was limited. Knee radiographs showed chondrocalcinosis and osteophytes, especially of the right knee (Figure 1). Twenty milliliters of milky pink synovial fluid were aspirated. After sedimentation, a white supernatant appeared (Figure 2). Polarized light microscopic examination showed fat globules without crystals or white blood cells (Figure 3). Measuring of lipids in the fluid confirmed high levels of fats (triglycerides $2.1 \mathrm{mmol} / \mathrm{L}$, total cholesterol $3.9 \mathrm{mmol} / \mathrm{L}$, and chylomicrons). Without further intervention or treatment, the pain and swelling improved over the next months and did not reoccur.

In making a diagnosis, the purulent, however milky, aspect of the synovial fluid may lead to a suspicion of septic arthritis with subsequent antibiotic treatment, whereas chylous joint effusions often have a benign course without treatment. ${ }^{3}$ Microscopy of the fluid is key to be able to differentiate the nature of the swollen joint.

\section{REFERENCES}

1. Reginato AJ, Feldman E, Rabinowitz JL. Traumatic chylous knee effusion. Ann Rheum Dis 1985;44:793-7.

2. White RE, Wise CM, Agudelo CA. Post-traumatic chylous joint effusion. Arthritis Rheum 1985;28:1303-6.

3. Soojian MG, Tejwani N. Chylous knee effusion: is it septic arthritis? A case report and review of literature. J Trauma 2004;57:1121-4.

4. Tahara M, Katsumi A, Akazawa T, Otsuka Y, Kitahara S. Posttraumatic chylous knee effusion. Knee 2011;18:133-5.

5. Borges PE, Franco MC, Recuero S, Sánchez-Pernaute O. Chylous joint effusion: a rare entity that mimics septic arthritis. Case Rep Intern Med 2015;2:13-7.

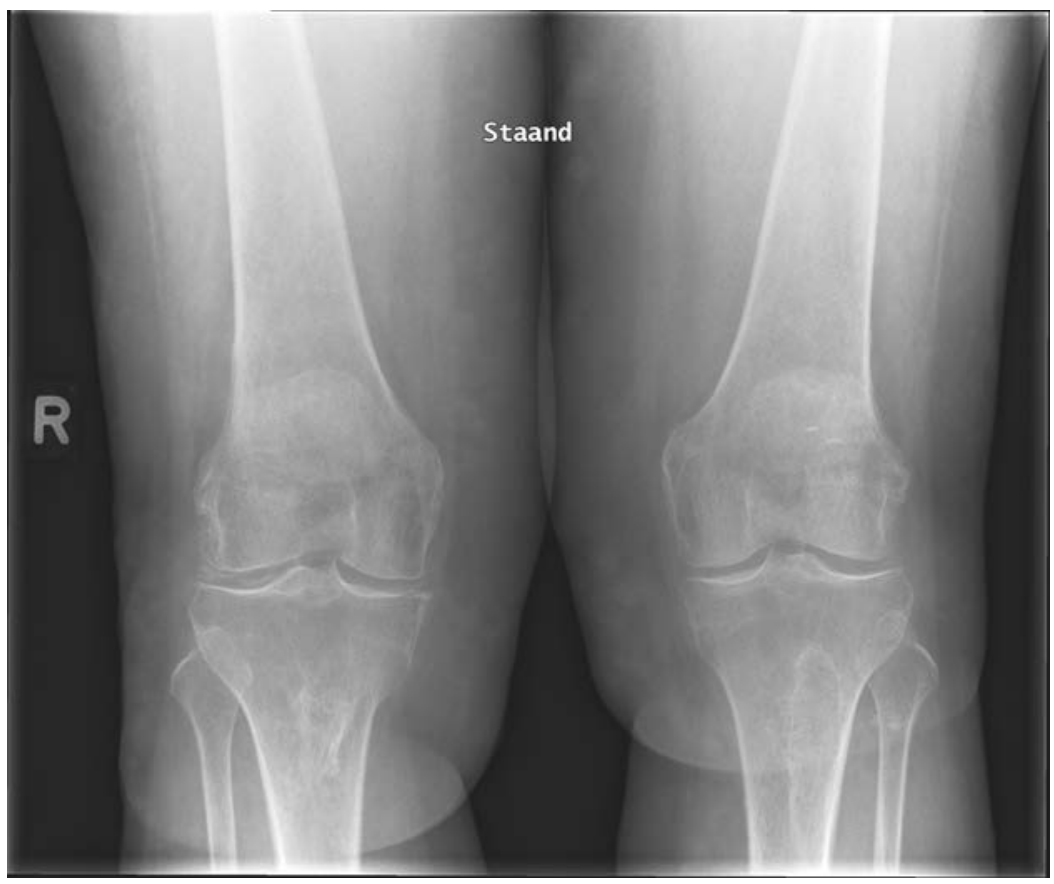

Figure 1. Radiograph of the knees. 


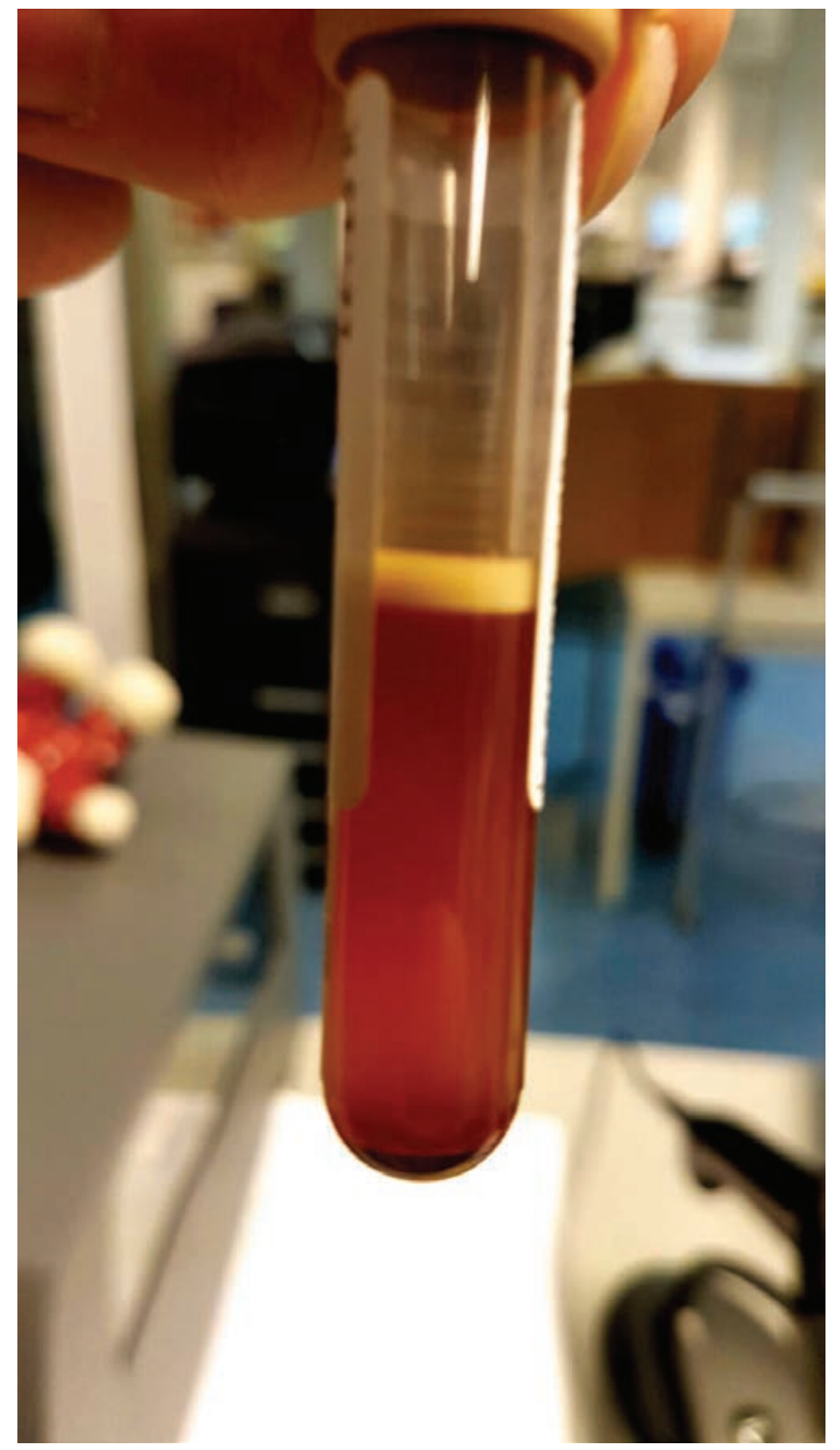

Figure 2. White supernatant after sedimentation.

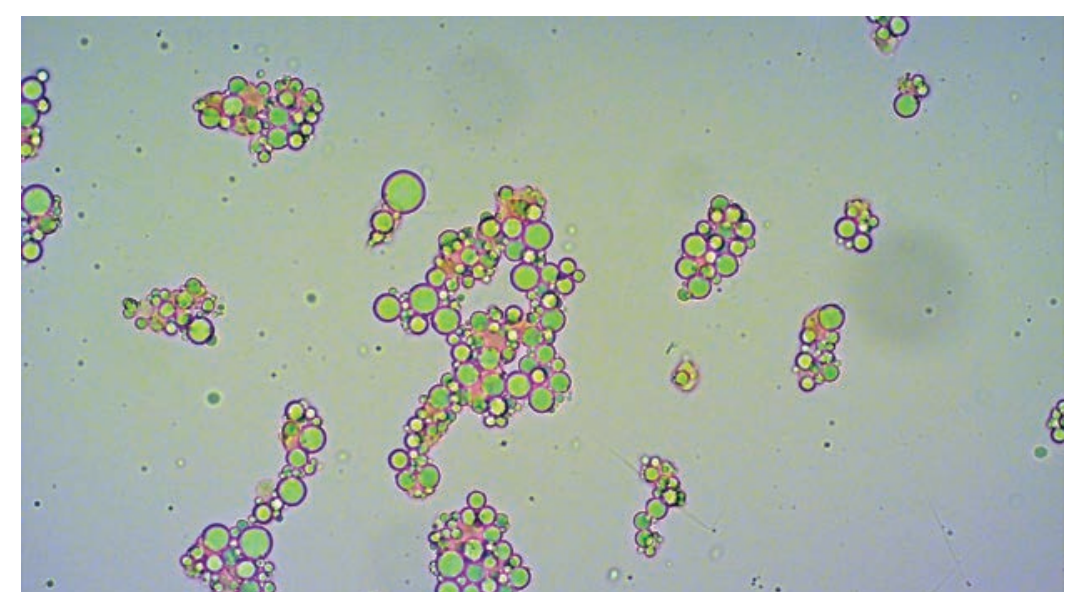

Figure 3. Polarized light microscopic examination. 